\title{
Avaliação da Ansiedade de Estudantes de Psicologia em Situações Experimentais de Falar em Público
}

\author{
Antonio Paulo Angélico ${ }^{1}$ \\ ${ }^{1}$ Universidade Federal de São João del-Rei, MG, Brasil.
}

\author{
Murilo Freitas Bauth ${ }^{2}$ \\ ${ }^{2}$ Centro Universitário de Lavras, $M G$, Brasil.
}

Resumo: Durante o percurso no ensino superior, os universitários se deparam com diversas atividades acadêmicas de falar em público (como, por exemplo, apresentações de seminários), que podem ser fortes causadores de ansiedade e afetos negativos, implicando prejuízos significativos para o rendimento acadêmico dos alunos e seus respectivos contextos sociais. Objetivou-se comparar grupos com e sem plateia em relação ao grau de ansiedade vivenciada frente a uma situação experimental de falar em público. Participaram 72 estudantes de psicologia, que foram submetidos ao Teste de Simulação de Falar em Público e responderam ao Inventário de Ansiedade de Beck, Inventário de Habilidades Sociais, Escala de Auto-Avaliação ao Falar em Público e Questionário Sociodemográfico e Ocupacional. Verificou-se que o grupo com plateia apresentou uma redução significativa do nível de ansiedade geral, subjetiva e autonômica após o discurso. Além disso, constatou-se que quanto mais elaborado o repertório de habilidades sociais gerais e de falar em público de um universitário e mais positivas as autoavaliações frente a essa tarefa, menor foi o grau de ansiedade experimentado nessa situação. Sugerem-se novas pesquisas com maior número de universitários, provenientes de diferentes cursos universitários, que permitam examinar as associações entre habilidades sociais, autoavaliações ao falar em público e ansiedade social.

Palavras-chave: Falar em Público, Avaliação do Desempenho, Interação Social, Autoavaliação.

\section{Evaluation of Anxiety in Experimental Situations of Public Speaking with Psychology Students}

\begin{abstract}
Throughout the course of higher education, college students are faced with a variety of academic public speaking activities (such as seminar presentations), which can be strong causes of anxiety and negative feelings, leading to significant damage to their academic performance and social contexts. It was aimed to compare groups with and without audience as to the level of anxiety experienced facing an experimental situation of public speaking. 72 psychology students were submitted to the Public Speaking Simulation Test and answered the Beck's Anxiety Inventory, Social Skills Inventory, Public Self Statements During Public Speaking Scale and Sociodemographic and Occupational Questionnaire. It was verified that the group with the audience presented a significant reduction of the level of general, subjective and autonomic anxiety after the speech. Besides, it was found that the more elaborate the general social skills and public speaking repertoire of a student were, as well as more positive self-assessments facing this task, the lower the degree of anxiety undergone in this situation. Further research is suggested with higher number of students, from different courses, allowing the examination and associations between social skills, selfassessment when public speaking and social anxiety.
\end{abstract}

Keywords: Public Speaking, Performance Evaluation, Social Interaction, Self-Evaluation 


\title{
Evaluación de la Ansiedad de Estudiantes de Psicología en Situaciones Experimentales de Hablar en Público
}

\begin{abstract}
Resumen: Durante su camino en la enseñanza superior, los estudiantes universitarios enfrentan a diversas actividades académicas de hablar en público (como las presentaciones de seminarios), que pueden provocar ansiedad y afectos negativos, causándoles pérdidas significativas en el rendimiento académico y sus respectivos contextos sociales. Se objetivó comparar grupos con y sin audiencia en relación con el grado de ansiedad experimentado frente a una situación experimental de hablar en público. Participaron 72 estudiantes de psicología, que realizaron la Prueba de Simulación de Hablar en Público y respondieron al Inventario de Ansiedad de Beck, el Inventario de Habilidades Sociales, la Escala de Autoevaluación al Hablar en Público y el Cuestionario sociodemográfico y ocupacional. Se verificó que el grupo con audiencia presentó una reducción significativa del nivel de ansiedad general, subjetiva y autonómica después del discurso. Además, se constató que cuanto más elaborado el repertorio de habilidades sociales generales y de hablar en público de un universitario y más positivas las autoevaluaciones frente a esa tarea, menor fue el grado de ansiedad experimentado en la situación. Se sugieren nuevas investigaciones con más universitarios de diferentes cursos para examinar las asociaciones entre habilidades sociales, autoevaluaciones al hablar en público y ansiedad social.
\end{abstract}

Palabras clave: Hablar en Público, Evaluación del Desarrollo, Interacción Social, Autoevaluación.

\section{Introdução}

Falar em público é considerado um poderoso estressor psicossocial que desencadeia altos níveis de ansiedade e afetos negativos (Osório, Crippa, \& Loureiro, 2008). Além disso, o comportamento de falar em público é um dos desempenhos sociais que mais exigem competência do indivíduo, visto que, independentemente da relevância do discurso proferido, sua qualidade dependerá da apresentação realizada (A. Del Prette \& Del Prette, 2001).

Segundo Pull (2012), indivíduos que possuem medo de falar em público podem apresentar prejuízos significativos em contextos sociais e ocupacionais. Estudos epidemiológicos evidenciaram que esse tipo de medo apresenta uma alta prevalência na população geral (D’el Rey \& Pacini, 2005; Stein, Walker, \& Forde, 1996) e, em particular, entre universitários (Baptista, 2007). Por exemplo, Marinho, Medeiros, Gama e Teixeira (2017) avaliaram a prevalência do medo de falar em público de 1.135 universitários de uma universidade pública de Belo Horizonte (MG). Foi observado que $63,9 \%$ da amostra relatou possuir medo de falar em público, sendo esse medo mais prevalente em mulheres (72,5\%), nos estudantes que participavam de poucas atividades de fala em grupo $(84,8 \%)$ e naqueles que autopercebiam suas vozes como negativas, com altura de voz para o agudo e volume de voz baixo $(68,7 \%)$. Além disso, por meio de uma escala que examinava as autoavaliações ao falar em público (Self Statements During Public Speaking Scale - SSPS), foi verificado que as mulheres apresentavam mais pensamentos negativos frente a essa situação quando comparadas aos homens.

Durante o percurso na universidade, os universitários entram constantemente em contato com atividades acadêmicas de falar em público (Baptista et al., 2012), como apresentações de trabalho em sala de aula e exposições de opinião em grupo (Landim et al., 2000). Essas tarefas podem ser fortes causadores de ansiedade e, assim, resultar em comportamentos de fuga e esquiva (por exemplo, faltar às aulas e recusar participar das atividades) que afetam o rendimento acadêmico desses indivíduos (Oliveira \& Duarte, 2004), ocasionando reprovações, dúvidas quanto à escolha profissional e evasão escolar. Ademais, estudantes que possuem déficits de habilidades de falar em público apresentam um maior risco de ter sua saúde prejudicada (Zimbardo, 1982) e, com o objetivo de aliviarem a ansiedade 
proveniente dessas tarefas, podem aumentar o consumo de álcool (Burke \& Stephens, 1999; Carrigan \& Randall, 2003; Gilles, Turk, \& Fresco, 2006) e medicamentos ansiolíticos, como, por exemplo, diazepam (Chutuape \& Wit, 1995).

De maneira geral, a ansiedade excessiva resultante do comportamento de falar em público pode ocorrer devido à falta de preparo e familiaridade com a tarefa (Clevenger \& Phifer, 1959) e cognições negativas (Puteri \& Fakhrurrozi, 2007). De acordo com os modelos cognitivos, o medo de falar em público advém tanto de uma avaliação negativa do indivíduo sobre o próprio desempenho quanto das expectativas de consequências negativas frente a essas tarefas, tais como a desaprovação da plateia quanto ao discurso proferido (Lopez, 1986), além de possíveis reações fisiológicas que podem interferir em seu desempenho - por exemplo, tremores, sudorese, gagueira, taquicardia, rubor, relaxamento dos esfíncteres, falhas na memória ("branco"), entre outras manifestações (Oliveira \& Duarte, 2004).

Em um estudo realizado por Schulz, Alpers e Hofmann (2008), 36 universitários com altos níveis de ansiedade social, de ambos os gêneros e com idade média de 18,83 anos $(D P=0,94)$, foram randomicamente designados para uma de duas condições antecipatórias de uma tarefa de falar em público. Na primeira condição, foram induzidas cognições negativas nos participantes, solicitando que eles se lembrassem de situações particulares em que não se saíram bem e qual foi a impressão que causaram nas outras pessoas nessas ocasiões. Baseados nessa imagem do passado, os participantes deveriam imaginar como poderiam desempenhar seu discurso, o que poderia dar errado e o que de pior aconteceria caso passassem vergonha. Na segunda condição, por sua vez, os estudantes eram submetidos a um estado de relaxamento, no qual ouviam uma gravação que os instruía a fecharem os olhos e se focarem em pensamentos, sentimentos e imagens agradáveis. Além disso, nessa situação, era requisitado que eles se livrassem de qualquer pensamento negativo que viesse em suas mentes.

Com relação aos resultados, Schulz et al. (2008) verificaram que a ansiedade autorrelatada e as cognições negativas aumentaram significativamente da linha de base para a situação de antecipação ao falar em público nos participantes da primeira condição, e reduziram para os participantes da segunda condição. De acordo com os autores, os altos níveis de ansiedade vivenciados foram mediados pela quantidade de autoavaliações negativas durante as condições de antecipação do discurso, reforçando o modelo cognitivo da ansiedade ao falar em público.

Em uma amostra de 81 estudantes de psicologia, de ambos os gêneros e com idade média de 19,86 anos ( $D P=1,39$ ), Weeks e Zoccola (2015) constataram que cognições relacionadas ao medo de avaliações negativas prediziam o aumento nos níveis de ansiedade frente a tarefas de falar em público. Em outro estudo realizado por Puteri e Fakhrurrozi (2007) com 50 universitários de ambos os gêneros, com idades variando de 18 a 22 anos, foi observado que os estudantes que mudaram suas cognições negativas, tornando-as positivas, apresentavam melhoras significativas nos níveis de ansiedade frente à situação de falar em público.

No mercado de trabalho, diversas profissões exigem que o indivíduo, pelo menos em algumas oportunidades, fale em público (Jones, Fazio, \& Vasey, 2012). Nesse sentido, considerando que os estudantes universitários se caracterizam por ser uma classe profissional emergente e que possuem, em algumas áreas de sua atuação, seja como objeto, seja como objetivo, a interação social (A. Del Prette, Del Prette, \& Castelo Branco, 1992; Z. A. P. Del Prette, Del Prette, \& Castelo Branco, 1992), há, em contrapartida, alta prevalência do medo de falar em público nessa população (Baptista, 2007; Marinho et al., 2017) e, devido a esse cenário, destaca-se a importância social e educacional de se avaliar o repertório de habilidades sociais de falar em público e seu referido medo nessa população.

Os fatores associados à experiência de falar em público são extensamente investigadosem populações com transtorno de ansiedade social (TAS) (Harb, Eng, Zairder, \& Heimberg, 2003; Laukka et al., 2008) e com altos níveis de ansiedade social (Schulz et al., 2008; Homer, Deeprose, \& Andrade, 2016). Outros estudos investigaram ainda as diferenças entre grupos com e sem TAS (Angélico, Crippa, \& Loureiro, 2012; Levitan et al., 2012; Voncken \& Bögels, 2008) e com diferentes níveis de ansiedade social (Cody \& Teachman, 2011; Sluis, Boschen, Neumann, \& Murphy, 2017; Wong \& Moulds, 2011). Por outro lado, observa-se na literatura a ausência de estudos que tenham investigado o medo de falar em público em populações não diagnosticadas com transtornos psiquiátricos ou não classificadas em condições subclínicas, como, por exemplo, pessoas com níveis altos de ansiedade social, mas que não satisfazem aos critérios diagnósticos para o TAS. 
Frente às considerações apresentadas, a presente pesquisa teve como objetivos: a) verificar possíveis diferenças entre os grupos com e sem plateia em relação aos níveis de ansiedade subjetiva experimentada frente a uma situação experimental de falar em público; e b) verificar as associações entre as manifestações subjetivas e comportamentais de ansiedade apresentadas pelos participantes diante da situação experimental. Para o desenvolvimento deste estudo, foi adotada a perspectiva cognitivo-comportamental.

\section{Método}

\section{Participantes}

Deste estudo, participaram 72 estudantes universitários, na faixa etária entre 17 e 30 anos $(M=21$ anos; $D P=2,73$ ), pareados conforme o gênero, regularmente matriculados no curso de psicologia de uma universidade pública do interior do estado de Minas Gerais, e designados em dois grupos, a saber: grupo com plateia e grupo sem plateia. O grupo com plateia foi composto por 32 universitários (16 do gênero feminino e 16 do masculino), com idade média de 21,7 anos $(D P=2,90)$, variando de 18 a 30 anos, e $9,4 \%$ dos quais exercendo alguma atividade profissional $(n=3)$. Compuseram o grupo sem plateia 40 universitários (20 do gênero feminino e 20 do masculino), com idade média de 20,4 anos $(D P=2,50)$, variando de 17 a 27 anos, e $12,5 \%$ dos quais exercendo alguma atividade profissional $(n=5)$. Da amostra total, apenas $11,11 \%$ participantes trabalhavam e estudavam.

Este estudo foi devidamente submetido à Comissão de Ética em Pesquisa Envolvendo Seres Humanos da universidade para sua apreciação e consequente aprovação (Memo no 067/2011-UFSJ/ CEPES). Todos os universitários que aceitaram participar do estudo assinaram o Termo de Consentimento Livre e Esclarecido.

\section{Instrumentos e materiais}

Neste estudo, foram utilizados os seguintes instrumentos: Inventário de Ansiedade de Beck (BAI), Inventário de Habilidades Sociais (IHS-Del-Prette), Escala de Auto-Avaliação ao Falar em Público (Self Statements During Public Speaking Scale - SSPS), Protocolo de Registro do Falar em Público (PRFP), além da situação experimental do falar em público, denominada Teste de Simulação de Falar em Público
(TSFP). Esses instrumentos e situação experimental serão descritos a seguir.

Inventário de Habilidades Sociais (IHS-DelPrette): é um instrumento de autorrelato, proposto por Z. A. P. Del Prette e Del Prette (2001), para a avaliação das dimensões situacional e comportamental molar das habilidades sociais, contendo 38 itens, cada um deles descrevendo uma relação interpessoal e uma possível reação àquela situação. Nas instruções, solicita-se que o respondente estime a frequência com que reage da forma sugerida em cada item, considerando o total de vezes que se encontrou na situação descrita e anotando sua resposta em escala Likert, variando de nunca ou raramente a sempre ou quase sempre. Seguem descritos, a seguir, os dados iniciais de validade e fidedignidade do IHS-Del-Prette (Z. A. P. Del Prette \& Del Prette, 2001), obtidos por Z. A. P. Del Prette, Del Prette e Barreto (1998) e Bandeira, Costa, Del Prette, Del Prette e Gerk-Carneiro (2000).

No estudo original, a análise das propriedades psicométricas com universitários obteve os seguintes resultados: índices positivos de discriminação dos itens, variando de 3,0 a 16,7; índices positivos de correlação item-escore total, com apenas dois índices não significativos e dez abaixo de 0,30 ; consistência interna satisfatória com um alfa de Cronbach de 0,75; e estrutura fatorial com cinco fatores principais, avaliada pelo método Alfa com rotação Varimax, cujos coeficientes alfa variaram de 0,74 a 0,96 , explicando $92,75 \%$ da variância total. Esses fatores foram denominados: enfrentamento e autoafirmação com risco (Fator 1), autoafirmação na expressão de sentimento positivo (Fator 2), conversação e desenvoltura social (Fator 3), autoexposição a desconhecidos e situações novas (Fator 4) e autocontrole da agressividade (Fator 5) (Del Prette et al., 1998). A fidedignidade foi avaliada por meio do método teste e reteste, obtendo-se um coeficiente de correlação de 0,90 ( $p=0,001)$ (Bandeira et al., 2000).

Self Statements During Public Speaking Scale (SSPS): instrumento autoaplicável, traduzido e adaptado para o português do Brasil por Osório et al. (2008), passando a se chamar Escala para Auto-Avaliação ao Falar em Público. Composta por dez itens, essa escala visa mensurar a autopercepção do desempenho na situação específica de falar em público. Em suas instruções, solicita-se que o respondente imagine as coisas que ele costuma pensar sobre si mesmo, quando se encontra em alguma situação em que tenha de falar em público, considerando até que ponto concorda 
com as situações e anotando suas respostas em escala Likert, variando de 0 (discordo totalmente) a 5 (concordo inteiramente).

$\mathrm{O}$ instrumento possui qualidades psicométricas satisfatórias, em termos de fidedignidade e validade. A correlação dos itens com o escore total variou entre 0,44 e 0,71 . A validade de construto foi estudada por meio da análise da estrutura fatorial, realizada pela técnica de Análise dos Componentes Principais com rotação Varimax. Foram extraídos dois fatores que corresponderam conjuntamente a $52 \%$ da variância dos dados (Osório et al., 2012). A consistência interna apresentou um alfa de Cronbach de 0,90 para a escala total, 0,80 para a subescala de autoavaliação positiva (Fator 1) e 0,78 para a subescala de autoavaliação negativa (Fator 2). Para esta última subescala, a pontuação dos itens é invertida, ou seja, quanto menor a média da pontuação, maior a avaliação negativa que o indivíduo tem de si (Osório et al., 2008).

Inventário de Ansiedade de Beck (BAI): instrumento autoadministrado composto por 21 itens que avaliam a gravidade dos sintomas de ansiedade, os quais são pontuados em uma escala Likert de 0 (absolutamente não) a 4 (gravemente). É subdividido em quatro subescalas: neurofisiológica, subjetiva, de pânico e autonômica. Foi utilizado em um estudo normativo com pacientes psiquiátricos e não psiquiátricos, incluindo neste grupo os estudantes universitários. O BAI demonstrou fidedignidade (alfa de Cronbach) de 0,93 para a amostra de pacientes identificados com transtorno de ansiedade social (TAS) e de 0,87 para a amostra de universitários; validade convergente de $0,78(p<0,001)$ com a subescala AnsiedadeEstado do Inventário de Ansiedade Traço-Estado (IDATE) (Spielberger, Gorsuch, \& Lushine, 1970); validade de construto em amostra de pacientes com TAS de $0,36(p<0,01)$ e $0,59(p<0,01)$ em amostra de universitários (Cunha, 2001).

Teste de Simulação de Falar em Público (TSFP): desenvolvido por McNair et al. (1982) e modificado por Guimarães, Zuardi e Graeff (1988), consiste em solicitar ao sujeito que prepare um discurso e o apresente a seguir em frente a uma câmera de vídeo que estará gravando seu desempenho.

Protocolo de Registro do Falar em Público (PRFP), adaptado de Angélico (2009): as categorias criadas para o protocolo de registro foram definidas e agrupadas em três classes de marcadores comportamentais de ansiedade, a saber: a) marcadores verbais (conteúdo negativo, repetição de conteúdo, repetição de palavra $\mathrm{e}$ uso de jargões); b) marcadores paralinguísticos (pausas silenciosas, pausas preenchidas, velocidade da fala inapropriada e intensidade de fala inapropriada); e c) marcadores não verbais, que compreendem as posturas corporais e os gestos apresentados pelos participantes (evitação da tarefa, rigidez corporal, inquietação, barreiras e latência de resposta). Para a observação dos marcadores verbais e paralinguísticos, foi empregada a técnica de registro de evento por fração de minuto, com intervalos de 30 segundos; e para a observação dos marcadores não verbais, a técnica de registro a intervalos de 15 segundos.

Visando a consecução dos objetivos desta pesquisa, além desses instrumentos, foram empregados, durante o período de coleta de dados, os seguintes equipamentos e materiais: uma câmera digital, uma televisão, um computador e DVDs. Para a análise dos dados, foram utilizados: televisão, DVD Player, computador, DVDs com as filmagens do TSFP e PRFP.

\section{Procedimentos}

Oito assistentes, previamente treinados, compuseram a equipe desta pesquisa. A coleta de dados foi realizada em duas etapas, a primeira com os participantes do grupo com plateia e a segunda com os integrantes do grupo sem plateia. Em cada etapa, um assistente de pesquisa conduziu o TSFP e outro, a aplicação das escalas. O conteúdo das filmagens foi analisado pelos assistentes de pesquisa, visando ao cálculo das frequências ou a duração dos marcadores comportamentais de ansiedade e dos índices de concordância entre observadores, a partir da seguinte fórmula:

Índice de Concordância $(\mathrm{IC})=\frac{\text { concordâncias }}{\text { concordâncias }+ \text { discordâncias }} \times 100$

Aos participantes, foi solicitado o comparecimento ao Laboratório de Ensino de Psicologia Experimental (Lapex) da universidade, em dia e horário previamente agendados, de acordo com a disponibilidade individual, para que fossem submetidos a uma situação experimental considerada um teste de ansiedade. Na ocasião da aplicação do teste, primeiramente, foram explicados os objetivos e procedimentos do estudo aos participantes. Em seguida, entregava-lhes o Termo de Consentimento Livre e Esclarecido (TCLE) para que o lessem e assinassem, caso concordassem em participar. 
Posteriormente, os sujeitos preencheram o Questionário Sociodemográfico, IHS-Del-Prette, SSPS e BAI, sendo este respondido antes e após a tarefa de falar em público. O procedimento completo de coleta de dados durou aproximadamente uma hora.

Com relação ao TSFP, o participante ficava em frente a uma câmera de vídeo e assistia a um videotape pré-gravado com instruções sobre a tarefa que teria de desempenhar. Recebia a seguinte instrução: "Você terá dois minutos para preparar um discurso, de quatro minutos, sobre sistema de cotas na universidade, que será gravado e, posteriormente, analisado por um psicólogo". Era computado o tempo de dois minutos para a preparação do discurso e, ao término, solicitado ao participante que o iniciasse. Transcorrido os quatro minutos, solicitava-se que permanecesse no ambiente, procurando relaxar, encerrando-se a sessão experimental.

Esses procedimentos listados foram aplicados em duas fases distintas, descritas a seguir. Em uma primeira fase do estudo, os participantes, designados para o grupo com plateia, proferiram o discurso para uma audiência composta por quatro espectadores (assistentes de pesquisa). Na segunda, enquanto os participantes (grupo sem plateia) proferiam seu discurso frente à câmera, seus desempenhos eram simultaneamente transmitidos no monitor da televisão, posicionado também a sua frente, para que pudessem acompanhar as suas performances e, assim, aumentar a veracidade da situação, de acordo com McNair et al. (1982).

\section{Análise de dados}

Os dados obtidos para a análise do conteúdo das filmagens foram codificados manualmente e alocados em um banco de dados criado em uma planilha do pacote estatístico SPSS (Statistical Package for Social Sciences) para o Windows, versão 22.0. Em seguida, análises estatísticas foram conduzidas com esses dados, por meio dos testes descritivos de média e desvios padrão. Com exceção dos escores totais e dos fatores do IHS-Del-Prette, os dados relativos às demais 34 variáveis testadas demonstraram não seguir distribuição normal $(p<0,05)$. Sendo assim, foram empregados testes não paramétricos para todas as análises estatísticas deste estudo, adotando-se o nível de significância de $p<0,05$.

O procedimento de categorização e análise das sessões experimentais observou os passos relatados a seguir. A partir das filmagens de cada participante, uma série de ações foi conduzida, a saber: (a) verificaram-se as ocorrências dos marcadores comportamentais, denominados de categorias, registrando-se as suas frequências; e (b) computou-se a duração da latência para o participante iniciar o discurso. Todas essas ações foram realizadas utilizando-se o protocolo de registro. O conteúdo do discurso dos participantes foi transcrito manualmente e depois digitado para possibilitar análises e categorizações mais precisas dos marcadores verbais de ansiedade.

Foi verificada a equivalência dos grupos com e sem plateia em relação aos níveis de ansiedade, em termos dos escores total e dos fatores do BAI, que poderiam interferir, potencialmente, na interpretação dos resultados deste estudo. Para esta análise, foi utilizado o teste de Mann-Whitney.

Empregou-se o teste deWilcoxon para comparar os níveis de ansiedade experimentados pelos grupos com e sem plateia antes e após o discurso (momento 1-M1 e momento $2-\mathrm{M} 2$, respectivamente). Para tal análise, foram utilizados os escores total e dos fatores do BAI.

Análises de correlação bivariada de Spearman foram realizadas para investigar as relações dos escores total e dos fatores do BAI com os escores total e dos fatores do IHS-Del-Prette, as subescalas positivas e negativas da SSPS, os itens e escore geral das habilidades sociais de falar em público (HSFP), extraídos do IHS, os marcadores de ansiedade e suas respectivas classes. Em concordância com outro pesquisador, foram selecionados os itens do IHS que melhor se ajustassem à habilidade de falar em público, a saber: itens 9 ("Evito fazer exposições ou palestras a pessoas desconhecidas"), 11 (“Em uma sala de aula ou reunião, se o professor ou dirigente faz uma afirmação incorreta, eu expresso meu ponto de vista”), 14 (“Faço exposição [por exemplo, palestras] em sala de aula ou no trabalho, quando sou indicado[a]"), 16 ("Em um grupo de pessoas conhecidas, se não concordo com a maioria, expresso verbalmente minha discordância") e 29 (“Na escola ou no trabalho, quando não compreendo uma explicação sobre algo em que estou interessado(a), faço as perguntas que julgo necessárias ao meu esclarecimento"). Esses itens tiveram em comum o componente de falar e/ou se expor em público, alguns deles com possibilidade de rejeição, réplica ou de oposição por parte do(s) interlocutor(es). Nas análises conduzidas, esses itens foram também somados para se obter um escore geral referente à habilidade de falar em 
público. Nessas análises, foram consideradas apenas as correlações que demonstraram significância estatística e sentido de conteúdo.

No tocante à avaliação da ocorrência dos marcadores comportamentais de ansiedade e suas respectivas classes durante a tarefa de falar em público, foram computados índices de concordância (ICs) entre duplas de observadores formadas entre cinco assistentes de pesquisa, previamente treinados. Para os marcadores de ansiedade e suas respectivas classes, os índices obtidos variaram de $96,8 \%$ a $99,9 \%$. Caso os observadores obtivessem ICs inferiores a 85\%, assistiam conjuntamente ao desempenho do participante em questão e o analisavam para identificar e discutir possíveis divergências, visando chegar a um consenso sobre a ocorrência e a frequência de marcadores específicos.

\section{Resultados}

A Tabela 1 exibe os dados relativos à equivalência entre os grupos com e sem plateia quanto à variável ansiedade. Nessa tabela, estão dispostos os valores da média e desvio padrão dos níveis de ansiedade para cada grupo e do teste de Mann-Whitney.

Considerando que os participantes não sabiam para qual grupo seriam designados, os resultados apresentados mostram que os grupos eram equivalentes em relação à ansiedade. No que se refere à classificação dos níveis de ansiedade prescritos pelo BAI (Cunha, 2001), 31 indivíduos estavam situados na classificação mínima (43\%), 31 na leve (43\%), 9 na moderada $(12,5 \%)$ e 1 na grave $(1,4 \%)$.

A Tabela 2 apresenta os dados referentes ao efeito do tempo (M1 e M2) sobre os escores total e dos fatores do BAI para os grupos com e sem plateia. Para esta análise, foi conduzido o teste de Wilcoxon.

Tabela 1.

Equivalência dos grupos com plateia $(n=32)$ e sem plateia $(n=40)$ em relação aos níveis de ansiedade frente à tarefa de falar em público.

\begin{tabular}{|c|c|c|c|c|}
\hline \multirow{2}{*}{ Inventário de Ansiedade de Beck } & \multirow{2}{*}{$\begin{array}{l}\text { Com plateia } \\
\text { Média }(D P)\end{array}$} & \multirow{2}{*}{$\frac{\text { Sem plateia }}{\text { Média }(D P)}$} & \multirow{2}{*}{$\begin{array}{l}\text { U de Mann- } \\
\text { Whitney }\end{array}$} & \multirow{2}{*}{ Probabilidade } \\
\hline & & & & \\
\hline Escore total & $13,92(8,78)$ & $11,62(6,27)$ & 561,5 & $p=0,373^{(\mathrm{NS})}$ \\
\hline Subescala neurofisiológica & $2,98(2,97)$ & $2,31(2,41)$ & 577,0 & $p=0,469^{(\mathrm{NS})}$ \\
\hline Subescala subjetiva & $5,68(3,53)$ & $4,97(2,82)$ & 596,0 & $p=0,616^{(\mathrm{NS})}$ \\
\hline Subescala de pânico & $2,05(2,43)$ & $1,75(1,58)$ & 623,5 & $p=0,848^{(\mathrm{NS})}$ \\
\hline Subescala autonômica & $3,23(2,41)$ & $2,59(2,03)$ & 557,5 & $p=0,344^{(\mathrm{NS})}$ \\
\hline
\end{tabular}

Nota: $D P=$ desvio padrão; (NS) = diferença não significativa.

Tabela 2.

Comparação entre momento 1 (M1) e momento 2 (M2) do discurso em relação aos escores total e dos fatores do BAI para os grupos com plateia $(n=32)$ e sem plateia $(n=40)$.

\begin{tabular}{|c|c|c|c|c|c|}
\hline \multirow{2}{*}{ Condição } & \multirow{2}{*}{$\begin{array}{l}\text { Diferença entre M1 e M2 em relação aos } \\
\text { escores total e dos fatores do BAI }\end{array}$} & \multicolumn{4}{|c|}{ Amostra total } \\
\hline & & $\overline{\mathbf{x}} \mathbf{1}(D P)$ & $\overline{\mathbf{x}} \mathbf{2}(D P)$ & $z$ & $p$ \\
\hline \multirow{5}{*}{ 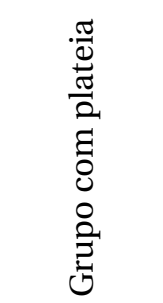 } & Escore total & $13,92(8,78)$ & $10,15(8,91)$ & $-2,478$ & $0,003^{*}$ \\
\hline & Subescala neurofisiológica & $2,98(2,97)$ & $2,65(3,15)$ & $-0,468$ & $0,405^{(\mathrm{NS})}$ \\
\hline & Subescala subjetiva & $5,68(3,53)$ & $3,98(3,53)$ & $-3,038$ & $0,005^{*}$ \\
\hline & Subescala de pânico & $2,05(2,43)$ & $1,72(1,68)$ & $-0,620$ & $0,525^{\text {(NS) }}$ \\
\hline & Subescala autonômica & $3,23(2,41)$ & $1,80(1,92)$ & $-2,438$ & $0,001^{*}$ \\
\hline
\end{tabular}

continua... 


\begin{tabular}{|c|c|c|c|c|c|}
\hline \multirow{2}{*}{ Condição } & \multirow{2}{*}{$\begin{array}{l}\text { Diferença entre M1 e M2 em relação aos } \\
\text { escores total e dos fatores do BAI }\end{array}$} & \multicolumn{4}{|c|}{ Amostra total } \\
\hline & & $\overline{\mathbf{x}} \mathbf{1}(D P)$ & $\overline{\mathbf{x}} \mathbf{2}(D P)$ & $z$ & $p$ \\
\hline \multirow{5}{*}{ 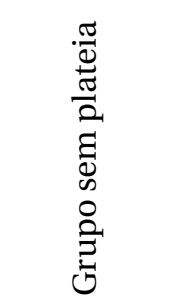 } & Escore total & $11,62(6,27)$ & $12,25(8,21)$ & $-0,141$ & $0,888^{(\mathrm{NS})}$ \\
\hline & Subescala neurofisiológica & $2,31(2,42)$ & $3,13(2,55)$ & $-1,692$ & $0,091^{(\mathrm{NS})}$ \\
\hline & Subescala subjetiva & $4,97(2,82)$ & $4,50(3,37)$ & $-1,197$ & $0,231^{(\mathrm{NS})}$ \\
\hline & Subescala de pânico & $1,75(1,59)$ & $1,72(1,61)$ & $-0,216$ & $0,829^{(\mathrm{NS})}$ \\
\hline & Subescala autonômica & $2,59(2,03)$ & $2,91(2,53)$ & $-0,462$ & $0,644^{(\mathrm{NS})}$ \\
\hline
\end{tabular}

Nota: $\mathrm{M} 1$ = momento $1 ; \mathrm{M} 2$ = momento $2 ; \overline{\mathrm{x}} 1$ = média do momento $1 ; \overline{\mathrm{x}} 2$ = média do momento $2 ; \mathrm{DP}=$ desvio padrão; * = diferença significativa; (NS) = diferença não significativa.

Na comparação entre M1 e M2 para o grupo com plateia, foram verificadas diferenças significativas no escore total e nas subescalas subjetiva e autonômica do BAI, indicando uma redução da ansiedade vivenciada nas três categorias apresentadas ao fim da tarefa de falar em público. Entretanto, no grupo sem plateia, não foram constatadas diferenças estatisticamente significativas nos níveis de ansiedade entre os dois momentos avaliados.
Os dados referentes aos escores total e dos fatores do BAI foram correlacionados com os escores total e dos fatores do IHS-Del-Prette, os itens relativos às habilidades sociais de falar em público e seu escore geral extraídos deste mesmo instrumento de medida, as subescalas positiva e negativa da SPSS e os marcadores comportamentais de ansiedade e suas respectivas classes. Os coeficientes de correlação de Spearman obtidos e os valores de $p$ correspondentes estão dispostos na Tabela 3 .

\section{Tabela 3}

Correlações significativas encontradas entre habilidades sociais gerais e de falar em público, ansiedade, autoavaliações e marcadores comportamentais de ansiedade na amostra do estudo ( $\mathrm{N}=72)$.

\begin{tabular}{lcc}
\hline \multicolumn{1}{c}{ Correlações significativas } & $\begin{array}{c}\boldsymbol{p d e} \\
\text { Spearman }\end{array}$ & Probabilidade \\
\hline Escore total IHS x escore total BAI & $-0,36$ & $p=0,002$ \\
Escore total IHS x subescala subjetiva BAI & $-0,35$ & $p=0,003$ \\
Escore total IHS x subescala de pânico BAI & $-0,29$ & $p=0,015$ \\
Escore total IHS x subescala autonômica BAI & $-0,25$ & $p=0,031$ \\
Fator 1 IHS x escore total BAI & $-0,26$ & $p=0,030$ \\
Fator 1 IHS x subescala subjetiva BAI & $-0,30$ & $p=0,010$ \\
Fator 3 IHS x escore total BAI & $-0,40$ & $p<0,001$ \\
Fator 3 IHS x subescala subjetiva BAI & $-0,29$ & $p=0,015$ \\
Fator 3 IHS x subescala de pânico BAI & $-0,30$ & $p=0,012$ \\
Fator 3 IHS x subescala autonômica BAI & $-0,45$ & $p<0,001$ \\
Fator 4 IHS x subescala de pânico BAI & $-0,25$ & $p=0,035$ \\
Item 9 IHS (Falar a público desconhecido) x subescala subjetiva BAI & $-2,32$ & $p=0,050$ \\
Item 9 IHS (Falar a público desconhecido) x subescala de pânico BAI & $-0,29$ & $p=0,015$ \\
\hline
\end{tabular}


...continuação

\section{Correlações significativas}

$p$ de
Spearman $\quad$ Probabilidade

Item 29 IHS (Fazer pergunta a conhecidos) x subescala subjetiva BAI

\begin{tabular}{rl}
$-0,24$ & $p=0,041$ \\
$-0,24$ & $p=0,039$ \\
$-0,28$ & $p=0,017$ \\
$-0,46$ & $p<0,001$ \\
$-0,50$ & $p<0,001$ \\
$-0,35$ & $p=0,002$ \\
$-0,40$ & $p=0,001$ \\
$-0,38$ & $p=0,001$ \\
$-0,26$ & $p=0,026$ \\
$-0,26$ & $p=0,031$ \\
0,28 & $p=0,016$ \\
$-0,26$ & $p=0,030$ \\
0,28 & $p=0,017$ \\
\hline
\end{tabular}

Escore geral HSFP x subescala subjetiva BAI

Escore geral HSFP x subescala de pânico BAI

Subescala negativa SSPS $\mathrm{x}$ escore total BAI

Subescala negativa SSPS $\mathrm{x}$ subescala subjetiva BAI

Subescala negativa SSPS x subescala de pânico BAI

Subescala positiva SSPS $\mathrm{x}$ escore total BAI

Subescala positiva SSPS $\mathrm{x}$ subescala subjetiva BAI

Subescala positiva SSPS x subescala de pânico BAI

Subescala positiva SSPS $\mathrm{x}$ subescala neurofisiológica BAI

Subescala subjetiva BAI x evitação de tarefa

Subescala de pânico BAI x latência de resposta

Subescala de pânico BAI x classe de marcadores não verbais

0,28

$p=0,017$

Nota: BAI: Inventário de Ansiedade de Beck; IHS: Inventário de Habilidades Sociais; Fator 1: enfrentamento e autoafirmação com risco; Fator 3: conversação e desenvoltura social; Fator 4: autoexposição a desconhecidos ou a situações novas;

HSFP: Habilidades Sociais de Falar em Público; SSPS: Self Statements During Public Speaking Scale.

Verificou-se que o escore total do BAI correlacionou-se negativa e significativamente com as duas subescalas da SSPS (positiva e negativa) e com o escore total e Fatores 1 e 3 do IHS-Del-Prette. Para a subescala subjetiva (BAI), foram encontradas correlações negativas e significativas com as duas subescalas da SSPS, os itens 9, 29, o escore geral da HSFP e o escore total e Fatores 1 e 3 do IHS. Além disso, essa mesma subescala correlacionou-se de maneira positiva e significativa com a evitação de tarefa.

No que diz respeito à subescala de pânico (BAI), constataram-se correlações positiva e significativa com a classe de marcadores não verbais, e negativas e significativas com as subescalas da SSPS, latência de resposta, com o item 9, o escore geral da HSFP e o escore total e Fatores 3 e 4 do IHS. Além disso, foram observadas correlações negativas e significativas entre a subescala autonômica e o escore total e Fator 3 do IHS e a subescala neurofisiológica com a subescala positiva da SSPS.

\section{Discussão}

O presente estudo teve como objetivo avaliar a ansiedade frente a uma tarefa de falar em público em universitários designados para duas condições experimentais (com e sem plateia) que, por sua vez, não foram submetidos à avaliação clínica. Devido à ausência de pesquisas que investiguem a ansiedade ao falar em público em populações não clínicas, serão discutidos a seguir resultados similares encontrados em outros estudos, que tangenciam os dados da presente pesquisa.

No que se refere à comparação entre os dois momentos da tarefa de falar em público sobre os níveis de ansiedade dos participantes, não foram constatadas diferenças significativas no grupo sem plateia entre os dois momentos avaliados. Entretanto, no grupo com plateia, a ansiedade geral, subjetiva e autonômica diminuíram significativamente ao final do discurso. Esses resultados foram similares aos encontrados por Chen, Mak e Fujita (2015), nos quais foi verificado que, ao fim de uma tarefa de falarem em público, com plateia, os estudantes apresentaram uma redução, embora não significativa, de seus níveis de ansiedade quando comparados ao início do discurso. De acordo com Savitsky e Gilovich (2003), mesmo aqueles indivíduos que não possuem medo acentuado de falar em público tendem a superestimar 
o quanto sua ansiedade é perceptível a uma audiência (ilusão de transparência), o que pode potencializar a ansiedade vivenciada e prejudicar o desempenho do discurso (Jones et al., 2012). Dessa forma, apesar de o grupo sem plateia deste estudo ter sido exposto a uma condição experimental, que, segundo McNair et al. (1982), aumenta a veracidade da situação de falar em público, acredita-se que o estímulo "presença de plateia" seja capaz de produzir mais ansiedade em indivíduos na referida tarefa; ao encerrarem seu contato com esse estímulo, os participantes do grupo com plateia podem ter vivenciado uma redução de seus efeitos ansiogênicos.

Concernente às correlações obtidas neste estudo, foi observado que: a) quanto mais elaborados são o repertório global de habilidades sociais e a classe de habilidades de conversação e desenvoltura social de um indivíduo, menor é a sensação de ansiedade geral, subjetiva, autonômica e de pânico vivenciada por ele; b) quanto mais desenvolvida é a classe de habilidades de enfrentamento e autoafirmação com risco, menores são os níveis de ansiedade geral e subjetiva vivenciados; c) quanto mais ricas são as habilidades de autoexposição a desconhecidos ou a situações novas de uma pessoa, menor é o grau de pânico experimentado; d) quanto mais elaboradas são as habilidades sociais de um indivíduo de falar em público, menor é o grau de ansiedade subjetiva e de pânico vivenciado; e) quanto mais positivas são as autoavaliações frente à situação de falar em público, menores são os níveis de ansiedade geral, subjetiva, neurofisiológica e de pânico vivenciados em um discurso; f) quanto mais negativas são as autoavaliações ao falar em público de uma pessoa, maior é o grau de ansiedade geral, subjetiva e de pânico experimentado por ela; g) quanto maior é o nível de ansiedade subjetiva experimentada pelo indivíduo, maior é a evitação da tarefa; e h) quanto maiores são os níveis de pânico vivenciados pela pessoa, menor é a latência de resposta e maior é a emissão da classe de marcadores não verbais.

Diante do exposto, verifica-se uma relação direta entre habilidades sociais, autoavaliações (cognições) ao falar em público e alguns marcadores comportamentais e sintomas de ansiedade. Dessa forma, pode-se afirmar que quanto mais elaborado for o repertório geral de habilidades sociais e de falar em público e mais positivas forem as cognições frente a essa tarefa, menor será a ansiedade experimentada nessas situações. De acordo com Caballo, Salazar, Irutia e OlivaresOlivares (2014), a correlação inversa entre habilidades sociais e ansiedade social pode ser compreendida a partir de duas possibilidades: a) o alto nível de ansiedade vivenciado por um indivíduo pode dificultar a emissão de seu repertório de habilidades sociais, ou b) a ausência de um repertório de habilidades sociais produz uma maior ansiedade vivenciada pelo indivíduo em situações sociais. Quanto às autoavaliações, os resultados encontrados se aproximam dos apresentados na literatura (Puteri \& Fakhrurrozi, 2007; Schulz et al., 2008; Weeks \& Zoccola, 2015), evidenciando a influência das cognições na ansiedade experimentada em tarefas de falar em público.

\section{Conclusões}

Em geral, foram demonstradas as associações entre a ansiedade e suas manifestações subjetivas (autoavaliações ao falar em público) e comportamentais apresentadas pelos universitários frente a uma situação experimental de simulação do falar em público. Além disso, como esperado, os grupos com e sem plateia diferiram significativamente em relação aos níveis de ansiedade ao final do discurso, sendo verificada uma dessensibilização ao efeito ansiogênico no primeiro grupo.

Embora exploratório, este estudo apresenta algumas limitações quanto aos procedimentos metodológicos utilizados. Além de não ter sido empregado um instrumento de medida que avaliasse especificamente a ansiedade social, esta pesquisa possuiu um número reduzido de sujeitos em cada grupo experimental, tendo sido realizada apenas com um curso de graduação, impossibilitando a generalização de resultados para outros cursos. Assim, recomenda-se a realização de novas pesquisas com a utilização de instrumentos de medida que avaliem a ansiedade social e uma amostra de universitários maior, provinda de diferentes cursos e áreas acadêmicas, que permitam a generalização dos dados obtidos e o exame da associação entre habilidades sociais, autoavaliações ao falar em público e ansiedade social. 


\section{Referências}

Angélico, A. P. (2009). Transtorno de ansiedade social e habilidades sociais: Estudo psicométrico e empírico (Tese de doutorado). Universidade de São Paulo, Ribeirão Preto, SP. http://www.teses.usp.br/teses/disponiveis/17/17148/ tde-02112009-151551/pt-br.php

Angélico, A. P., Crippa, J. A. S., \& Loureiro, S. R. (2012). Transtorno de ansiedade social e habilidades sociais de falar em público: Estudo experimental. Estudos e Pesquisas em Psicologia, 12(1), 10-35. http://pepsic.bvsalud.org/pdf/ epp/v12n1/v12n1a02.pdf

Bandeira, M., Costa, M. N., Del Prette Z. A. P., Del Prette, A., \& Gerk-Carneiro, E. (2000). Qualidades psicométricas do Inventário de Habilidades Sociais (IHS): Estudo sobre a estabilidade temporal e validade concomitante. Estudos de Psicologia (Natal), 5(2), 401-419. https://doi.org/10.1590/S1413-294X2000000200006

Baptista, C. A. (2007). Estudo da prevalência do transtorno de ansiedade social em estudantes universitários (Dissertação de mestrado). Universidade de São Paulo, São Paulo, SP. http://pgsm.fmrp.usp.br/wp-content/ uploads/2011/11/MESTRADO-CARLOS-ALBERTO-BAPTISTA.pdf

Baptista, C. A., Loureiro, S. R., Osório, F. de L., Zuardi, A. W., Magalhães, P. V, Kapczinski, F., Santos Filho, A., Freitas-Ferrari, M. C., \& Crippa, J. A. S. (2012). Social phobia in Brazilian university students: Prevalence, under-recognition and academic impairment in women. Journal of Affective Disorders, 136(3), 857-861. https://doi.org/10.1016/j.jad.2011.09.022

Burke, R. S., \& Stephens, R. S. (1999). Social anxiety and drinking in college students: A social cognitive theory analysis. Clinical Psychology Review, 19(5), 513-530. https://doi.org/10.1016/S0272-7358(98)00058-0

Caballo, V. E., Salazar, I., Irurtia, M. J., \& Olivares-Olivares, P. J. (2014). Relación de las habilidades sociales con la ansiedad social y los estilos/trastornos de la personalidad. Behavioral Psychology/Psicologia Conductual, 22(3), 401-422. https://www.researchgate.net/publication/269762295_Relacion_de_las_habilidades_sociales_con_la_ ansiedad_social_y_los_estilostrastornos_de_la_personalidad

Carrigan, M. H., \& Randall, C. L. (2003). Self-medication in social phobia: A review of the alcohol literature. Addictive Behaviors, 28(2), 269-284. https://doi.org/10.1016/S0306-4603(01)00235-0

Chen, J., Mak, R., \& Fujita, S. (2015). The effect of combination of video feedback and audience feedback on social anxiety: Preliminary findings. Behavior Modification, 39(5), 721-739. https://doi.org/10.1177/0145445515587087

Chutuape, M. A., \&Wit, H. (1995). Preferences for ethanol and diazepam in anxious individuals: An evaluation of the self-medication hypothesis. Psychopharmacology, 121, 91-103. https://doi.org/10.1007/BF02245595

Clevenger, T. J., \& Phifer, G. (1959). What do beginning college speech texts say about stage fright? The Speech Teacher, 8(1), 1-10. https:// doi.org/10.1080/03634525909376987

Cody, M. W., \& Teachman, B. A. (2011). Global and local evaluations of public speaking performance in social anxiety. Behavior Therapy, 42(4), 601-611. https://doi.org/10.1016/j.beth.2011.01.004

Cunha, J. A. (2001). Manual da versão em português das Escalas Beck. São Paulo, SP: Casa do Psicólogo.

Del Prette, A., \& Del Prette Z. A. P. (2001). Psicologia das relações interpessoais: Vivências para o trabalho em grupo. Petrópolis, RJ: Vozes.

Del Prette, A., Del Prette, Z. A. P., \& Castelo Branco, U. V. (1992). Competência social na formação do psicólogo. Paidéia: Cadernos de Psicologia e Educação, 2, 40-50. https://doi.org/10.1590/S0103-863X1992000200005

Del Prette, Z. A. P., Del Prette, A., \& Castelo Branco, U. V. (1992). Competência social: Um levantamento de situações críticas de interação para estudantes de psicologia. Anais do I Congresso Nacional de Psicologia Escolar, Campinas, SP, 1, 384-387. https://bit.ly/3pdab53

Del Prette, Z. A. P., \& Del Prette, A. (2001). Inventário de Habilidades Sociais (IHS-Del-Prette): Manual de aplicação, apuração e interpretação. São Paulo, SP: Casa do Psicólogo.

Del Prette, Z. A. P., Del Prette, A., \& Barreto, M. C. M. (1998). Análise de um inventário de habilidades sociais (IHS) em uma amostra de universitários. Psicologia: Teoria e Pesquisa, 14(3), 219-228. https://revistaptp.unb.br/index.php/ ptp/article/view/1507/468

D’el Rey, G. J. F., \& Pacini, C. A. (2005). Medo de falar em público em uma amostra da população: Prevalência, impacto no funcionamento pessoal e tratamento. Psicologia: Teoria e Pesquisa, 21(2), 237-242. https://doi.org/10.1590/ S0102-37722005000200014 
Gilles, D. M., Turk, C. L., \& Fresco, D. M. (2006). Social anxiety, alcohol expectancies, and self-efficacy as predictors of heavy drinking in college students. Addictive Behaviors, 31(3), 388-398. https://doi.org/10.1016/j.addbeh.2005.05.020

Guimarães, F. S., Zuardi, A. W., \& Graeff, F. G. (1988). Effect of chlorimipramine and maprotiline on experimental anxiety in humans. Journal of Psychopharmacology, 1(3), 184-192. https://doi.org/10.1177/026988118700100305

Harb, G. C., Eng, W., Zaider, T., \& Heimberg, R. G. (2003). Behavioral assessment of public-speaking anxiety using a modified version of the Social Performance Rating Scale. Behaviour Research and Therapy, 41(11), 1373-1380. https://doi.org/10.1016/S0005-7967(03)00158-X

Homer, S. R., Deeprose, C., \& Andrade, J. (2016). Negative mental imagery in public speaking anxiety: Forming cognitive resistance by taxing visuospatial working memory. Journal of Behavior Therapy and Experimental Psychiatry, 50, 77-82. https:// doi.org/10.1016/j.jbtep.2015.05.004

Jones, C. R., Fazio, R. H., \&Vasey, M.W. (2012). Attentional control buffers the effect of public speaking anxiety on performance. Social Psychological and Personality Science, 3(5), 556-561. https:// doi.org/10.1177/ 1948550611430166

Landim, A. K. P., Costa, C. F., Conilheiro, D., Sá, E. S., Nunes, E. C., Santos, E. E., Batista, E. A. R., Lima, F., Santos, G. S., Rego, S. C. O. B., Souza, S. F., \& Martinez, A. (2000). Ansiedade social em estudantes universitários: Preocupações mais emergentes. Revista Unicastelo, 3(3), 185-192.

Laukka, P., Linnman, C., Åhs, F., Pissiota, A., Frans, Ö., Faria, V., \& Furmark, T. (2008). In a nervous voice: Acoustic analysis and perception of anxiety in social phobics' speech. Journal of Nonverbal Behavior, 32(4), 195-214. https://doi.org/10.1007/s10919-008-0055-9

Levitan, M. N., Falcone, E. M., Placido, M., Krieger, S., Pinheiro, L., Crippa, J. A., \& Nardi, A. E. (2012). Public speaking in social phobia: A pilot study of self-ratings and observers' ratings of social skills. Journal of Clinical Psychology, 68(4), 397-402. https://doi.org/10.1002/jclp.20868

Lopez, A. B. (1986). Analisis de componentes de un tratamiento cognitivo-somatico-conductual del miedo de hablar en publico (Tese de doutorado). Universidade de Barcelona, Barcelona. https://www.tdx.cat/handle/10803/2547

Marinho, A. C. F., Medeiros, A. M., Gama, A. C. C., \& Teixeira, L. (2017). Fear of public speaking: Perception of college students and correlates. Journal of Voice, 31(1), 127.e7-127.e11. https://doi.org/10.1016/j.jvoice.2015.12.012

McNair, D. M., Frankenthaler, L. M., Czerlinsky, T., White, T. W., Sasson, S., \& Fisher, S. (1982). Simulated public speaking as a model of clinical anxiety. Psychopharmacology, 77(1), 7-10. https://doi.org/10.1007/BF00436092

Oliveira, M. A., \& Duarte, Â. M. M. D. (2004). Controle de respostas de ansiedade em universitários em situações de exposições orais. Revista Brasileira de Terapia Comportamental e Cognitiva, 6(2), 183-199. http://www.usp.br/rbtcc/index.php/RBTCC/article/view/56/45

Osório, F. L., Crippa, J. A. S., \& Loureiro, R. S. (2008). Escala para auto-avaliação ao falar em público (SSPS): Adaptação transcultural e consistência interna da versão brasileira. Revista de Psiquiatria Clínica, 35(6), 207-211. https://doi.org/10.1590/S0101-60832008000600001

Osório, F. L., Crippa, J. A. S., \& Loureiro, S. R. (2012). Aspectos cognitivos do falar em público: Validação de uma escala de autoavaliação para universitários brasileiros. Revista de Psiquiatria Clínica, 39(2), 48-53. https://doi.org/10.1590/S0101-60832012000200002

Pull, C. B. (2012). Current status of knowledge on public-speaking anxiety. Current Opinion, 25(1), 32-38. https://doi.org/10.1097/YCO.0b013e32834e06dc

Puteri, N. D., Fakhrurrozi, M (2007). The relationship between positive mindset and anxiety in public speaking of students. Depok: Gunadarma University. http://202.125.94.81/abstract/graduate/1243-3803-1-SM.pdf

Savitsky, K., \& Gilovich, T. (2003). The illusion of transparency and the alleviation of speech anxiety. Journal of Experimental Social Psychology, 39(6), 618-625. https://doi.org/10.1016/S0022-1031(03)00056-8

Schulz, S. M., Alpers, G. W., \& Hofmann, S. G. (2008). Negative self-focused cognitions mediate the effect of trait social anxiety on state anxiety. Behaviour Research and Therapy, 46(4), 438-449. https://doi.org/10.1016/j.brat.2008.01.008

Sluis, R. A., Boschen, M. J., Neumann, D. L., \& Murphy, K. (2017). Anticipatory processing in social anxiety: Investigation using attentional control theory. Journal of Behavior Therapy and Experimental Psychiatry, 57, 172-179. https://doi.org/10.1016/j.jbtep.2017.05.009

Spielberger, C. D., Gorsuch, R. L., \& Lushine, R. E. (1970). Manual for the state-trait anxiety inventory. Palo Alto: Consulting Psychologists. 
Stein, M. B., Walker, J. R., \& Forde, D. R. (1996). Public-speaking fears in a community sample: Prevalence, impact on functioning and diagnostic classification. Archives of General Psychiatry, 53(2), 169-174. https://doi.org/10.1001/archpsyc.1996.01830020087010

Voncken, M. J., \& Bögels, S. M. (2008). Social performance deficits in social anxiety disorder: Reality during conversation and biased perception during speech. Journal of Anxiety Disorders, 22(8), 1384-1392. https://doi.org/10.1016/j.janxdis.2008.02.001

Weeks, J. W., \& Zoccola, P. M. (2015). "Having the heart to be evaluated": The differential effects of fears of positive and negative evaluation on emotional and cardiovascular responses to social threat. Journal of Anxiety Disorders, 36, 115-126. https:// doi.org/10.1016/j.janxdis.2015.08.004

Wong, Q. J. J., \& Moulds, M. L. (2011). Behaviour research and therapy impact of anticipatory processing versus distraction on multiple indices of anxiety in socially anxious individuals. Behaviour Research and Therapy, 49(10), 700-706. https:// doi.org/10.1016/j.brat.2011.07.007

Zimbardo, P. G. (1982). A timidez. Lisboa: Edições70.

\section{Antonio Paulo Angélico}

Professor Associado do Departamento de Psicologia/ Programa de Pós-Graduação em Psicologia/ Universidade Federal de São João del-Rei, São João del-Rei - MG. Brasil.

E-mail: angelico.fmrp.usp@gmail.com

(1) https://orcid.org/0000-0002-6926-0439

\section{Murilo Freitas Bauth}

Professor Adjunto do Departamento de Psicologia do Centro Universitário de Lavras, Lavras - MG. Brasil.

E-mail: murilobauth.psi@gmail.com

(1) https://orcid.org/0000-0003-4003-9498

Pesquisa parcialmente financiada pela Fundação de Amparo à Pesquisa do Estado de Minas Gerais (FAPEMIG Processo no. APQ-04059-10).

Endereço para envio de correspondência:

Universidade Federal de São João del-Rei. Praça Dom Helvécio, 74, Dom Bosco, CEP: 36301-160 - São João

del-Rei - MG. Brasil.

Recebido 18/09/2018

Aceito 06/04/2020

Received 09/18/2018

Approved 04/06/2020

Recibido 18/09/2018

Aceptado 06/04/2020 
Como citar: Angélico, A. P., \& Bauth, M. F. (2020). Avaliação da Ansiedade de Estudantes de Psicologia em Situações Experimentais de Falar em Público. Psicologia: Ciência e Profissão, 40, 1-14.

https://doi.org/10.1590/1982-3703003214267

How to cite: Angélico, A. P., \& Bauth, M. F. (2020). Evaluation of Anxiety in Experimental Situations of Public Speaking with Psychology Students. Psicologia: Ciência e Profissão, 40, 1-14.

https://doi.org/10.1590/1982-3703003214267

Cómo citar: Angélico, A. P., \& Bauth, M. F. (2020). Evaluación de la Ansiedad de Estudiantes de Psicología en Situaciones Experimentales de Hablar en Público. Psicologia: Ciência e Profissão, 40, 1-14.

https://doi.org/10.1590/1982-3703003214267 